\title{
A Contribution for the Diagnosis of Insulation Cables due different conditions of degradation, voltage stress and frequency.
}

\author{
F. N. Lima ${ }^{1}$, Dr.; R. W. D. R. França ${ }^{1}$, Eng; A. P. Finazzi ${ }^{1}$, Dr.; B. C. Carvalho ${ }^{1}$, Dr.; \\ Ariela Zanoni Conejo ${ }^{1}$, Acad.; Marina Timo de Sá ${ }^{1}$, Acad; Iago de Moura Faria ${ }^{1}$, Eng. \\ ${ }^{1}$ Federal University of Mato Grosso (UFMT), Department of Electrical Engineering - Cuiabá (Brazil) \\ e-mail: fernandn@ufmt.br, kingrapa@gmail.com, apfinazzi@ufmt.br, bcc@ufmt.br, arielaconejo@ gmail.com, \\ marinatimosa@hotmail.com, iago_mf@hotmail.com
}

\begin{abstract}
It is widely recognized that one of the major problems responsible for unscheduled interruptions is related to the degradation of power cables insulation. The water tree effect emerges as an important cause for the deterioration of their insulation layer. In this context, the loss current technique is a suitable method for investigating slight degradation, since the cable degradation by water tree gives rise to harmonics components in the loss current. This paper presents an analysis of the influence of the length of the water-tree, the voltage stress and the frequency in the values of various parameters that has been used to evaluate, predict, and indicate the operational status of operating power cables. The procedure aims to estimate the parameters of insulation deterioration from the measurement of the supply voltage and the leakage current that flows through the insulation carried out in hot-line cables without interrupting the service.
\end{abstract}

\section{Key words}

Insulated cable, water tree, loss current, cable diagnosis, power system.

\section{Introduction}

Questions related to power quality definitely challenge engineers all over the world. Amongst these, the matter of energy supply reliability emerges as an important point towards the improvement of continuity indexes such as the duration and frequency of interruptions. In this way, the information associated to the operational condition of equipment such as transformers, power cables, have a relevant role in the behavior of the electrical system. The reference [1] reported that many factors could result in the formation of faults in power cables and accessories. These could form in the process of manufacturing, handling, storage, transportation and installation.

Among the most prominent phenomena responsible for the degradation of power cables, the water tree [2-5] consists in a relatively common problem that is developed in the insulation layer of these components. Usually, it occurs when power cable is immersed in humid environment and presents voids and impurities inside the insulation or protrusions in the semiconductor layer of XLPE cables.

Two types of trees are developed which are vented water tree which develops in cable screen as shown in Fig. 1, and bow tie tree which develops in cable insulation $[4,5]$.

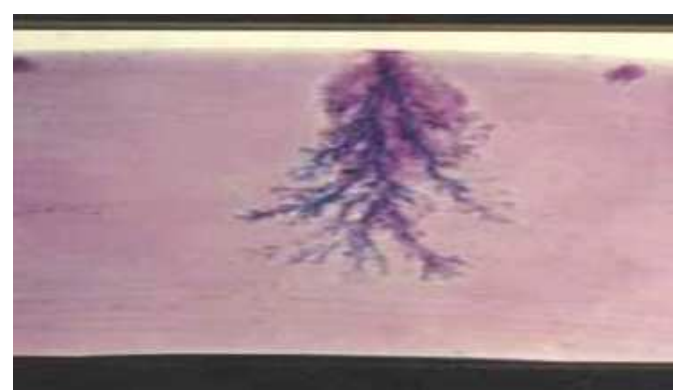

Fig. 1. Vented water tree in the cable screen [4]

The Fig. 2 shows the physical conception and allows noting two different and complementary circuits. The first one is destined to represent the healthy portion of the cable (multiple RC sets combined in a parallel association). The second one is composed by a series combination of a nonlinear resistance that represents the insulation damage, and a linear capacitor aiming to indicate the healthy regions around the water tree deterioration. Naturally, the values assigned to these parameters will dictate the level of degradation involved in each situation under analysis.

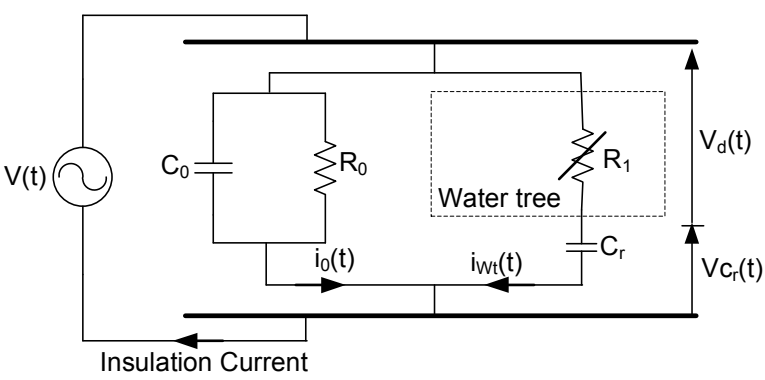

Fig. 2. Simplified equivalent circuit which comprises healthy and degraded parts, for a given length of cable.

In Figure 2:

- $\mathrm{C}_{0}$ - Equivalent capacitance of the non-degraded cable extension;

- $\mathrm{R}_{0}$ - Equivalent resistance of the non-degraded cable extension;

- $\mathrm{C}_{\mathrm{r}}-$ Equivalent capacitance of the residual non-degraded region along the deteriorated cable insulation extension;

- $\mathrm{R}_{1}$ - Equivalent resistance of the degraded region along the deteriorated cable insulation extension; 
Aiming at the diagnosis of the operational conditions of an insulated cable, in terms of life expectance, several methods have been developed and employed to evaluate, predict, and indicate the operational status of operating cables. In particular, when seeking practical ways for determining the final effects of water tree phenomenon on cable degradation, several researches aims to propose procedures to detect this kind of failure through the extraction of information from performance parameters that indicate the degradation level of the insulation layer of a power cable and assist in deciding about the replacement of the cables. Some methods examine the component in a de-energized condition while others are based on measurements carried out with an energized cable but without load. The reference [6] describes a synthesis of these strategies.

The present paper is inserted in the context of the diagnostic method utilizing the harmonics in the loss current as a technique to diagnose water tree deterioration in XLPE cables. The loss current relates to power losses in the insulation. As water tree degradation advances, the loss current waveform becomes distorted and harmonic components are included as a result of the nonlinear voltagecurrent characteristic of the water tree. The evaluation of these current harmonics provides a suitable diagnostic method for evaluating, predicting, and indicating the operational status of operating cables. The reference [7] presents a brief review on methods introduced in literature to utilize the loss current as a diagnostic tool to identify and quantify the power cable degradation.

The loss current technique is a diagnostic method that can be used to identify the un-bridged water tree, which can induce the sudden breakdown [8]. Then, for the high-voltage class power cable, an accurate detection of this un-bridged water tree becomes important due its higher operating stress.

The main objective of this study is to observe the behavior of a set of indicators for cables degraded by water tree, considering different voltage stress and frequency. The authors believe that the results will be useful in a diagnostic method to detect water tree at the early stages and to estimate the cables physical operational conditions.

\section{Performance parameters}

Cables that exhibit varied degrees of degradation have, as a particular characteristic, the leakage current waveform with a third harmonic dominant effect as well as a small level of direct current [9]. Based on these options, the work carried out under this research included the use of a set of degradation indexes [10] and new performance parameters, based on the nonlinear behavior of degraded insulators that are summarized next.

- Dielectric Power Losses - The power dissipated in the dielectric $(P)$ is a state indicator that is directly associated with the degree of losses found in the cable insulation and can be used to estimate the density of deterioration [11];

- Total Harmonic Distortion (THD) of loss current - This indicator shows increasing values as the degree of cable deterioration rises. Its values exhibit much stronger dependence with electric stress than the dielectric losses and a better correlation with long trees [11]. The $\tan \delta$ is an effective diagnostic technique in cases where deteriorations are uniform. Therefore, it cannot discriminate between the occurrence of numerous water trees with slight deteriorations, and a few water trees with serious deterioration [9].

- Third harmonic amplitude $\left(\mathrm{I}_{3}\right)$ of leakage current - The magnitude of the third order harmonic current existent in the leakage current, in accordance with [9], tends to grow as the degradation level increases. This indicator behaves similarly to the Individual Harmonic Distortion (IHD), however, it presents itself, as an alternative to solve the dispersion that can occur in the analysis, since the harmonic in question seems dominant;

- Third harmonic phase angle $\left(\theta_{3}\right)$ of loss current - It is a performance parameter, which has a good correlation with the water tree length, that decreases with the increase of the water tree length [11]. As the deterioration advances, the $\theta_{3}$ decreases, showing a trend to a fixed direction [9]. Moreover, there is an extremely good correlation of $\theta_{3}$ with the extent of development of water trees and degradation of the breakdown strength [10];

- Equivalent parameter - The indicator directly express the leakage resistances of the cable. When the deterioration progresses, the insulation resistance usually decreases [12] and the capacitance of the cable increases [13]. This behavior can be used as a signal of insulation deterioration in XLPE cables;

- Linearity indicator of $V$-I relationship - It is an indicator that relates the instantaneous values of applied voltage to the cable and their leakage currents, obtaining, consequently, correlations that express a larger or minor linearity relation. The Pearson Coefficient [14] is used to characterize the level of non-linearity existent in the measurements.

\section{Loss current measurement}

The loss current technique requires the identification of the loss current flowing in the insulation layer, which is used as reference to identify the degree of the cable insulation degradation. The Fig.3 shows one of the schematic diagram of the experimental which is used for identify the loss current [15].

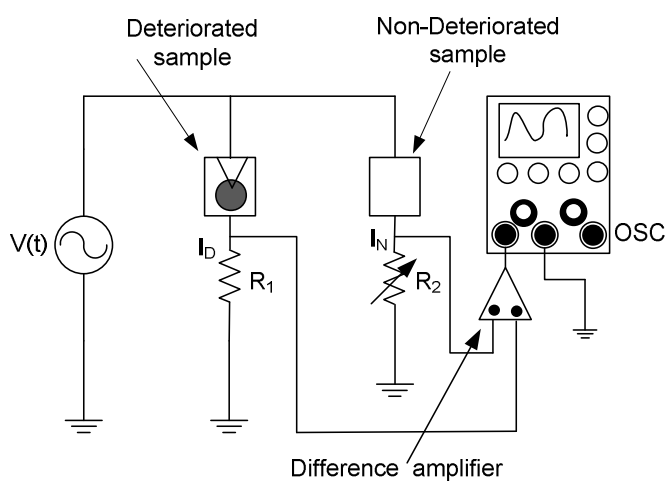

Fig. 3. Loss current measuring circuit [15]

The basic operating principle of the above scheme is described below. 
First, it is necessary to identify the currents flowing in each branch of the circuit, as follows: $\mathrm{I}_{\mathrm{N}}(\mathrm{t})$ corresponding to the non-degraded sample current, $I_{D}(t)$ corresponds to a degraded sample current and $\left[I_{N}(t)-I_{D}(t)\right]$ is the output current of the differential amplifier. The same sinusoidal voltage is applied to non-degraded and degraded samples. The voltages across the $R_{1}$ and $R_{2}$ resistors are connected to a differential amplifier. By observing the waveform current in the differential amplifier on an oscilloscope it is possible to identify the loss current by adjusting the resistance used for detecting the current through the non-degraded sample to make the difference current, $I_{N}(t)-I_{D}(t)$, in phase with the applied voltage [16].

This procedure does not consider the possibility of harmonic capacitive current, because only the fundamental frequency is considered in the capacitive current [17].

In reference [15] an equivalent model was validated by water tree degradation using this type of measurement circuit. Through this model, the authors identified some loss current curves in function of the length of the water tree, the stress voltage and frequency. These curves are used as reference in this case study to verify their influences in a set of parameters used to evaluate the operative condition of the insulation of insulated cables.

Therefore, it is required to eliminate the capacitive harmonic components from these current waveforms. The difficulty consists in estimating, for each case, what should be the true angle of the third harmonic of the loss current, which was overcome by determining the behaviour of this angle due to the percentage of nonlinearity, as will be presented in the following section.

\section{Theta 3 variation due the degradation}

There is evidence that the degradation increasing entails an increment of harmonics in the leakage current and causes the third angle harmonic to tend to zero, as can be seen in Fig. 4 [9].

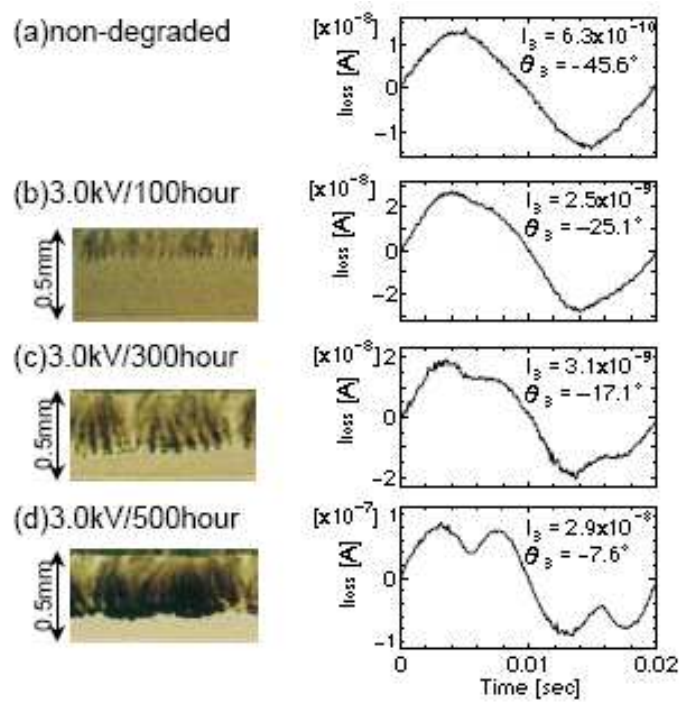

Fig. 4. Loss current waveforms: a) non-degraded; b) $3.0 \mathrm{kV} / 100$ hour; c) $3.0 \mathrm{kV} / 300$ hour and d) $3.0 \mathrm{kV} / 500$ hour [9]
The waveform presented in Fig. 4 was obtained from samples where water trees were grown using a liquid electrode composed by a $1-\mathrm{mol} / \mathrm{l} \mathrm{NaCl}$ solution placed at one side of a XLPE sheet sample $(0.5 \mathrm{~mm}$ thick), while a sinusoidal voltage of amplitude $3 \mathrm{kV}$ and frequency $1 \mathrm{~Hz}$ was applied during a specific time interval. A test sinusoidal voltage waveform of amplitude $1 \mathrm{kV}$ and frequency $50 \mathrm{~Hz}$ was used in the loss current measurement, which was obtained with a bridge circuit using a lossless standard capacitor connected in parallel with the cable sample by subtracting the currents through the capacitor and the sample [9].

Fig. 5 shows the $I-V$ characteristic curve for all aforementioned cases, obtained in [18]. It is possible to observe that the curve is nearly linear in cases where small degradation exists, thus implying that the insulation resistance is linear and no water trees exist.

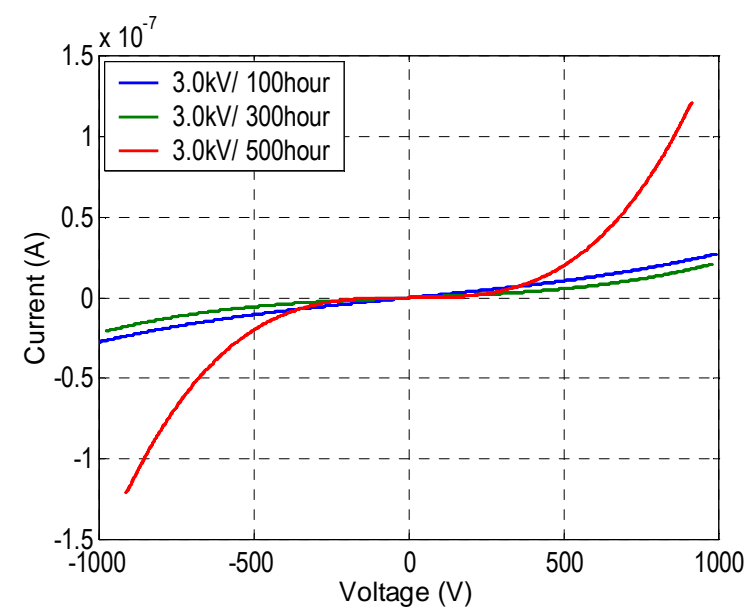

Fig. 5. $I-V$ characteristic curves of water trees [18]

Knowing the $I-V$ characteristics, it becomes possible to identify the behaviour of the third harmonic angle due to the advancement of degradation. To do so, the Non Linearity Coefficient of Pearson of characteristics shown in Fig.5 was used.

The Table I presents the Pearson coefficients modified of these characteristics, more precisely how this ratio departs from 1, plus two additional points, one being related to optimum insulation and the other for completely degraded layer associated with the respective third harmonic phase.

Table I. - $\theta_{3}$ behavior due to degradation
\begin{tabular}{|c|c|}
\hline$\%$ & $\theta_{3}$ \\
\hline 0 & -90 \\
\hline 1.5 & -45.6 \\
\hline 3.7 & -25.1 \\
\hline 17.8 & -17.1 \\
\hline 49.8 & -7.6 \\
\hline 100 & 0 \\
\hline
\end{tabular}

It is important to note that when this coefficient is equal to 1 means that the relationship between the two quantities is linear. As it moves away from 1 the relationship between these values becomes increasingly non-linear. When this coefficient is 1 means no 
degradation in the insulation and when it is zero the degradation is in the entire length of the insulation. That is, when the Pearson coefficient ranges from 1 to zero, theoretically the degradation will vary from $0 \%$ to $100 \%$. For clarity of numerical indicator, this degradation index will use a greatness that expresses the percentage difference between the measured value and the unitary reference $(\triangle \mathrm{CP} \%)$.

Finally considering that the third harmonic phase has a good correlation with water tree length [10] and correlating the water tree length with the level of degradation represented by the index degradation, it is possible to identify the relation between the third harmonic angle and the increasing degradation of the insulating layer shows, in the Fig. 6.

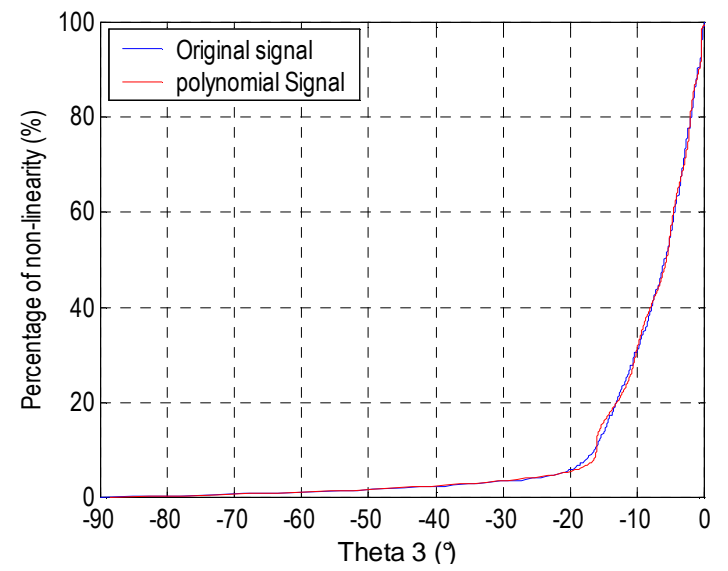

Fig. 6. $\theta_{3}$ variation due to degradation

This relation will be represented by a polynomial of order 11, to eliminate the capacitive harmonic components in the current as shows in the adopted reference in this paper [17]. To eliminate these harmonics components, the methodology presented in [18], will be useful in the current signal identification delay, which ensures the value of $\theta_{3}$ expected.

To examine the influence of the length of the water tree, the frequency variation and voltage stress from the information available in the reference used, it is adopted in this study, the hypothesis that the characteristic shown in Fig. 6, may be used in any case as long as there is information about the level of degradation of the insulating layer. Whereas if there is no information about the third harmonic of the loss current the current waveform presented in Fig. 4d will represent the degradation when the growth of the water tree is $90 \%$.

\section{Degradation influence in performance parameters}

As mentioned before, to assess the influence of the water tree length, the voltage stress and the frequency in a set of performance parameters, in order to diagnose the water tree in its initial stage the curves available in [15] will be used.

In the calculation of the performance parameters will be considered the fundamental and the 3rd, 5th and 7 th harmonics order of the loss current. a) Water tree length

The Fig. 7 shows the calculated loss current in various water tree lengths as a function of time. The applied voltage is $5 \mathrm{k} \mathrm{Vp}-\mathrm{p} 1 \mathrm{kHz}$.

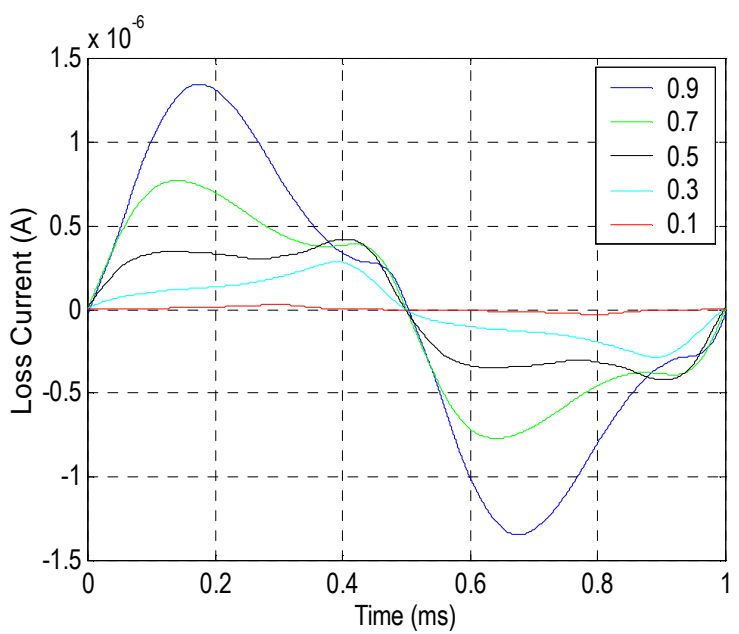

Fig. 7. Loss current waveform [15]

The Fig. 8 shows the influence of the degraded area on a set of performance parameters, determined from the loss current, after the elimination of the respective capacitive harmonic contributions. The parameters $\mathrm{P}, \mathrm{R}$ and $\mathrm{I}_{3}$ were multiplied by 20,2 and 60 , respectively.

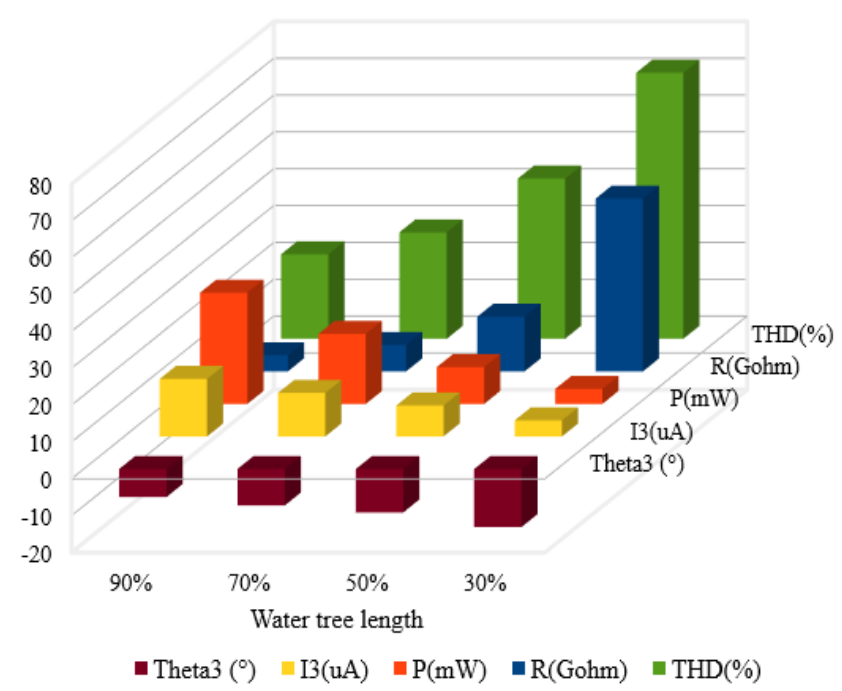

Fig. 8. Performance parameters due water tree length

The expansion of the degraded region leads to a decrease in resistance and consequently an increase in the loss current and the dissipated power. The phase angle $\theta_{3}$, which has a good correlation with the length of the tree, increase with degradation. These results are in accordance with the expected results. However, in the case of THD, it is observed that this performance parameter decreases with the increase degradation. It is possible that in all cases the non-linear characteristic of the water tree was maintained. Therefore, with the increase degradation the ratio between $I_{3}$ and $I_{1}$ decrease, which can be verified in the Fig. 9. 


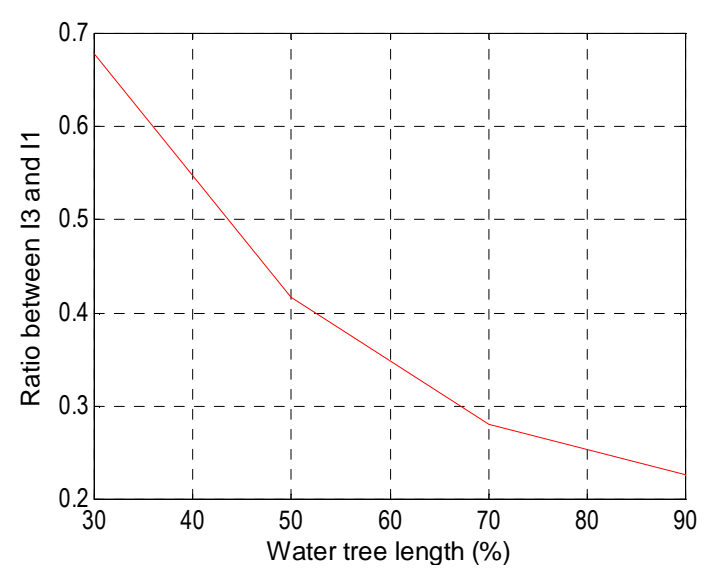

Fig. 9. Ratio between $I_{3}$ and $I_{1}$

\section{b) Stress Voltage}

Since finding the water tree at an initial stage is a purpose in [15], the length of water tree is assumed to be $30 \%$. The frequency of the applied voltage is also fixed by $1 \mathrm{kHz}$.

The Figure 10 shows the calculated loss current in various applied voltage.

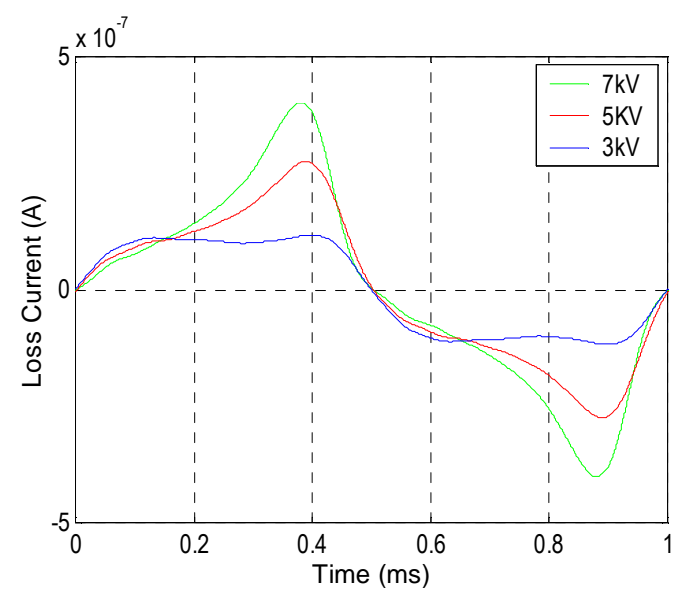

Fig. 10. Loss current waveform in various applied voltage [15]

The Fig. 11 shows the influence of voltage stress applied to a given level of water tree, in the performance parameters adopted in this study. The parameters $\theta_{3}, R$ and $I_{3}$ were multiplied by 2,10 and 800 , respectively.

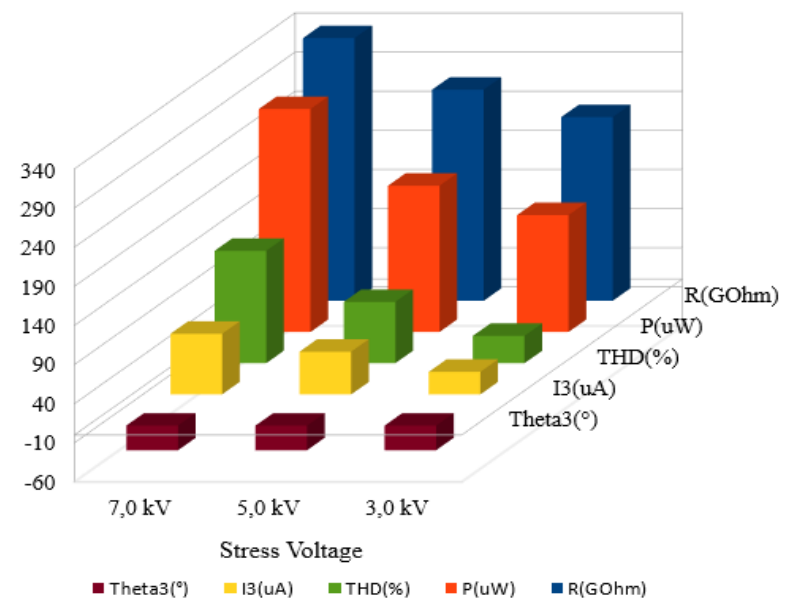

Fig. 11. Performance parameters for various stress voltage
Considering that the degradation is the same in length $(30 \%)$ and also in non-linearity of the $I-V$ characteristic of the water tree it is possible to conclude that the increase in voltage stress has the effect of increasing not only the loss current, but mainly increase the distortion of the resulting waveforms. This expansion of degradation would not be accentuated if the $I-V$ characteristic of the water tree was close to a straight line, which in practice represents insulation with low degradation. Furthermore, the behavior of phase angle $\theta_{3}$, indicates that it is a parameter related to the length of the degradation.

\section{c) Frequency}

The Fig. 12 shows the calculated loss current in various frequencies, because of this to plot in the same figure the frequencies were adjusted to be in the reference of $1 \mathrm{kHz}$. In this case, the applied voltage and the length of water tree are fixed by $5 \mathrm{kV}$ and $30 \%$ respectively. It is found that the values of loss current increase with the increase of the frequency [15]. The curves referring to $100 \mathrm{~Hz}$ and $1 \mathrm{kHz}$ were multiplied by 25 and 6 , respectively.

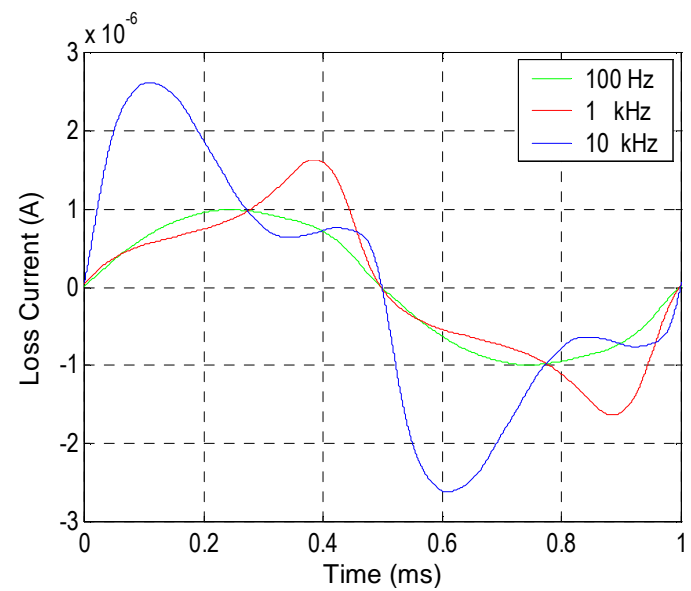

Fig. 12. Loss current waveform in various frequency [15]

The Fig. 13 shows the influence of the frequency on an insulation suggesting a certain level of degradation in the already mentioned parameters. The parameters THD and $\mathrm{I}_{3}$ were multiplied by 5 and 60 , respectively.

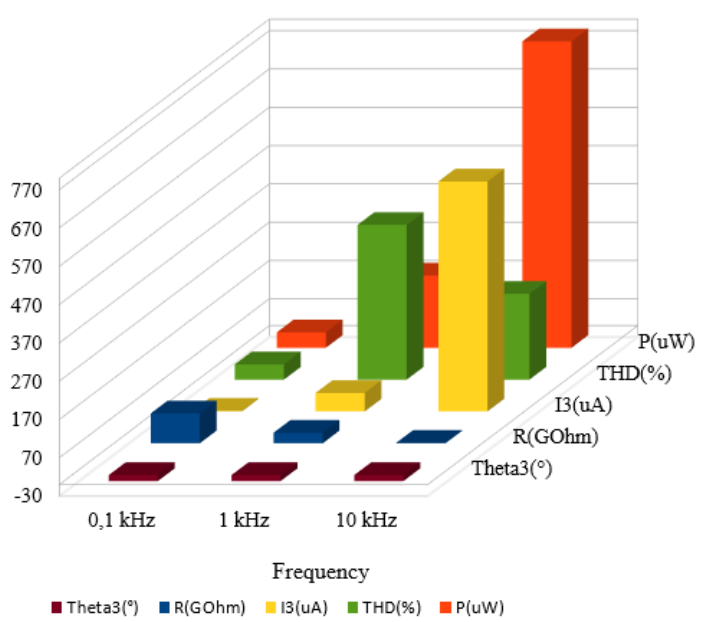

Fig. 13. Performance parameters in various frequency 
The frequency variation takes effect on the capacitive reactance. It is expected that the increased frequency entails an increase in the leakage current, justifying the increase in the fundamental and harmonic components of the loss current, and the decrease in the equivalent resistance. Because of this, the dielectric power losses increase. The results for the phase angle $\theta_{3}$ confirms that it is an important parameter for identifying the length of the degradation. However, for THD is required to compare $100 \mathrm{~Hz}$ and $10 \mathrm{kHz}$ with the reference of $1 \mathrm{kHz}$, where both had a smaller value. The first case was because of the reduction in the leakage current with the reduction in the harmonic distortion. The second case, appears to be mainly because the fundamental current presents a greater increase than the harmonic distortion in the loss current, making the THD decrease. It is important to note that, when the frequency of the applied voltage increase, the distortion of the leakage current increases because the capacitive harmonics had more influence than the active harmonics in the leakage current. For this reason, the THD of the leakage current is more indicated than the THD of the loss current to detect small degradations.

\section{Conclusions}

This investigation has presented a contribution to the diagnosis of operating conditions of insulated cables degraded by water trees. The methodology allows obtaining several indicators regarding water tree degradation, which can be obtained from the loss current that flows through the degraded sample. The results obtained from the parameters are in accordance with the literature and confirm the importance of the theoretical model used in this study for understanding and identifying the best way to detect water tree at early stages. Another relevant aspect concerns the possibility to identify, with a very good accuracy, the loss current only from the measured leakage current without the need for specific measurement circuit used to distinguish the loss current that flows in the insulation layer. One aspect to be considered in future studies relates to the influence of distorted supply voltages on the performance of the aforementioned performance parameters.

\section{References}

[1] IEEE Guide for Partial Discharge testing of Shielded Power Cables System in a Field Environment. (IEEE Std 400.3tm -2006).

[2] S. Priya and A. Mubashira Anjum, "Analysis of Water Trees and Characterization Techniques in Xlpe Cables", Indian Journal of Science and Technology, Vol. 7(S7), 127-135, ISSN: 0974-6846, November, 2014.

[3] Skoršepová Terézia, "The growth of water trees in XLPE cable's insulation", Journal Elektrotechnika, Ročník 6, číslo 9, Portál pre odborné publikovanie ISSN 1338-0087, Set, 2013.

[4] Aluru Divya Teja, K. Rajagopala, "Electric field effect in the formation of water treeing in MV power cables". IJRET: International Journal of Research in Engineering and Technology. Volume: 03 Issue: 04, Apr 2014.
[5] Che Nuru Sanniyati, Yanuar Z. Arief, Zuraimy Adzis, Nor Asiah Muhamad, Mohd. Hafizi Ahmad, Muhammad Abu Bakar Sidik, K. Y. Lau. "Water Tree in Polymeric Cables: A review". Malaysian Journal of Fundamental and Applied Sciences.Vol.11, Nº.4 (2015) 191-200, 2015.

[6] S. Yamaguchi, S. Soda, N. Takada, "Development of a New Type Insulation Diagnostic Method for Hot-Line XLPE Cables", IEEE Transactions on Power Delivery, July, Vol. $4 \mathrm{~N}^{\mathrm{o}} 3$, pp. 1513-1520, 1989.

[7] Hussain, N.; Nor, N.M. ; Abdullah, M.F. ; Ashraf, K.; Usman Baloch, M, "Review of Third Harmonic Current Characteristic based Diagnostic Methods of medium Voltage Power Cables Insulation". Sustainable Utilization and Development in Engineering and Technology (STUDENT), IEEE Conference on, 2012.

[8] T. Furuhashi, K. Tohyama, T. Imai, Y. Murata, "Dissipation Current Waveform of Water Tree Deteriorated Low Density Polyethylene Sheet". Electrical Insulation and Dielectric Phenomena, 2006 IEEE Conference on, Oct. 15-18, pp. 529-532, 2006.

[9] T. Tsujimoto, M. Nakade, Y. Yagi, K. Adachi, H. Tanaka, Egasakicho, Tsurumi-ku, Yokohama, Kanagawa, Yawata-kaigandori, Ichihara, Chiba, Higashi-shinagawa, Shinagawa-ku, "Development of on-site Diagnostic for XLPE Cable by Harmonics in AC Loss Current" Proceedings of the 7th International Conference on Properties and Applications of Dielectric Materials, June 1-5, Nagoya, pp 73-76, 2003.

[10] Y. Yukihiro., T. Hideo and K. Hitoshi., "Study on Diagnostic Method for Water Treed XLPE Cable by Loss Current Measurement," 0-7803-53035-9/98/\$10.00@ 1998 IEEE, pp. 653656,1998

[11] A. T. Bulinski, E. So, S. S.Bamji, "Measurement of the Harmonic Distortion of the Insulation Loss Current as a Diagnostic Tool for High Voltage Cable Insulation,” 0- 7803-5935-6/00/\$10.00 @ 2000 IEEE, pp. 1615-1620, 2000.

[12] Wei. Zhang, Yutao Zhau, Baitun Yang, Yaonan Liu, "A Study on DC Component Method for Hot-Line XLPE Cable Diagnosis," IEEE International Symposium on Electrical Insulation, Pittsburgh - PA USA, June 5-8, pp. 95- 98, 1994.

[13] T. Ozaki, N. Ito, I. Sengoku, J. Kawai, and S. Nakamura, "Changes of capacitance and dielectric dissipation factor of water-treed XLPE with voltage,", Electrical Insulating Material, 2001. Proceedings of 2001 International Symposium, Himeje, Japan, pp. 459-462, 2001.

[14] S. A. Lira, "Correlation Analysis: Theoretical Approach and Construction of Coefficients with Applications", Ms.C.Dissertation, Federal University of Parana, Curitiba, 2004.

[15] Suzuki, M., Itoh, A., Yoshimura, N., "Loss Current Analysis of Water Tree Degradation in Polyethylene using Equivalent Circuit Model", IEEJ Transactions on Fundamentals and Materials, Volume 125, Issue 4, pp. 367- 372, 2005.

[16] N. Ito, T. Ozaki, I. Sengoku, J. Kawai,"Relation between the AC loss current method and PEA method for water-treed length", IEEE Electrical Insulating Materials. Proceedings of 2001 International Symposium on, Himeji, Japan, pp. 524-527, Nov, 2001.

[17] Lima, F. N.; Finazzi, A. P.; Carvalho, B. C.; Faria, I. M.; Campos, G. P.. A Comparative Analysis of Loss Current Obtained by Measuring Circuits used in Studies of Degradation Power Cables. Renewable Energy \& Power Quality Journal (RE\&PQJ), v. 14, p. 301-306, 2016.

[18] F. N. Lima, J. C. Oliveira, D. A. Andrade, B. C. Carvalho, A. P Finazzi, R. M. T. Silva, "Modeling Water Tree Phenomenon for Insulated Cable Loss Current Estimation", Transmission and Distribution Conference and Exposition Latin America, 2008 IEEE/PES, August 13-15, Bogotá, Colombia, pp. 1-6, 2008. 